\title{
Modelling of anisotropic work-hardening behaviour of metallic materials subjected to strain-path changes
}

\author{
S. Bouvier ${ }^{a, *}$, J.L. Alves ${ }^{b}$, M.C. Oliveira ${ }^{\text {c }}$, L.F. Menezes ${ }^{c}$ \\ a LPMTM-CNRS UPR9001, Université Paris 13, 99, Av. J.B. Clément, 93430 Villetaneuse, France \\ ${ }^{\mathrm{b}}$ Department of Mechanical Engineering, University of Minho, Campus de Azurém, 4810 Guimarães, Portugal \\ ${ }^{\mathrm{c}}$ Department of Mechanical Engineering, University of Coimbra, Polo II, 3030 Coimbra, Portugal
}

\begin{abstract}
The present paper aims at reviewing some recent progress in developing advanced constitutive models which are devoted to the description of the anisotropic work-hardening behaviour under strain-path changes at large strains of metallic materials. After reviewing some microscopic and macroscopic experimental evidence, a physically-based phenomenological model using four internal state tensor variables is presented. This model can be simplified into several classical phenomenological models in order to take into account either the isotropic or the kinematic hardening or both. The implementation of the proposed models in the in-house finite element code DD3IMP is briefly recalled. Numerical simulations of the stamping of a curved rail are carried out in order to evaluate the accuracy and the efficiency of the proposed models in modelling the springback.
\end{abstract}

(c) 2004 Elsevier B.V. All rights reserved.

Keywords: Dislocation pattern; Microstructural heterogeneity; Anisotropic behaviour; Yield surface; Sheet metal forming; Finite element; Non-proportional loading

\section{Introduction}

Sheet metal forming processes may often involve intense forming sequences, leading to large strains and severe strain-path changes. Optimizing such technologies requires a good modelling of the

\footnotetext{
* Corresponding author. Tel.: +33149403478.

E-mail address: salima.bouvier@lpmtm.univ-paris13.fr (S. Bouvier).
}

anisotropic plastic behaviour of deformed materials. Different effects are related to the material such as its initial textural anisotropy, or its induced work-hardening anisotropy due to strainpath changes. Various types of models can be used to describe this initial and/or induced anisotropy, according to their required ability to explain and/ or predict the details of the plastic behaviour during a given deformation process. Thus, rather simple models of isotropic hardening can already give a sufficiently good estimate of the drawing 
or ironing forces occurring during the process. More sophisticated models, involving a non-linear kinematic hardening and internal state variables that describe the directional strength and the polarity of dislocation structures, are expected to allow an improved evaluation of the springback and of the residual stresses in the formed parts, as well as a better prediction of eventual strain localizations after strain-path changes.

The present paper aims at shortly reviewing a few advanced constitutive models to discuss their identification by mechanical tests, and to illustrate their ability to predict the plastic behaviour under strain-path changes and at large strains. The first section of this paper is devoted to a rapid reviewing of microscopic and macroscopic experimental evidence which can explain the macroscopic anisotropic behaviour of metallic materials. A physically-based phenomenological model using four internal state tensor variables is presented in the second section of this paper. This model includes more classical phenomenological models in order to take into account either the isotropic or the kinematic hardening or both. The third section describes some details of the implementation of the proposed models in the in-house finite element code DD3IMP. Numerical simulations of the deep drawing of a curved rail are performed in order to evaluate the accuracy and the efficiency of the proposed models in modelling the springback.

\section{Evolution of the intragranular microstructure}

In recent years, several microstructural studies by transmission electron microscopy [1-10], among others) were carried out in order to understand the anisotropic work-hardening behaviour of polycrystalline metals under strain-path changes at large strains. Most of the previous studies were devoted to a better knowledge of the relations between the microstructure evolution (i.e. dislocations organizations), the change of the crystallographic texture and the anisotropy of the macroscopic behaviour after two-stage loading. The latter is characterized by the parameter $\theta$ proposed by Schmitt et al. [11].
$\theta=\frac{\mathbf{D}_{1}: \mathbf{D}_{2}}{\left|\mathbf{D}_{1}\right| \cdot\left|\mathbf{D}_{2}\right|}$

In this expression, $\mathbf{D}_{1}$ and $\mathbf{D}_{2}$ represent the strain rate tensors during the prestrain and the subsequent deformation, respectively, and $\|\mathbf{D}\|$ is the norm of $\mathbf{D}$ defined by $\|\mathbf{D}\|=\sqrt{D_{i j} D_{i j}}$. The socalled quasi-monotonic, quasi-Bauschinger, and orthogonal strain-path changes are defined by $\theta=1,-1$ and 0 , respectively. From a microstructural point of view, a monotonic sequence corresponds to the activation of the same slip systems in the same direction before and after reloading, while a Bauschinger sequence to the activation of the same slip systems in opposite directions and finally an orthogonal sequence to the activation of different slip systems. These different sequences allow underlining the combined effects of the microstructural and textural evolutions on the macroscopic behaviour of materials.

The main conclusions of the previously referred to research can be summarized as follow:

- During proportional loading at room temperature and moderate and large strains of polycrystalline metals, several slip systems are generally activated simultaneously in most of the grains and the dislocations self organize in cells, creating cell walls with high dislocation density and cell interior with low density.

- Depending on the deformation mode, and the crystallographic orientation, either planar parallel dislocation walls or closed walls of more equiaxed cells form at moderately large strains (Figs. 1 and 2), the latter process leading generally to a refinement or subdivision of the grains. Furthermore, the orientation and the size of the dislocation walls depend on the loading path, as well as on the amount of strain. Many studies suggest that this substructure affects the flow stress evolution under strain-path changes, such as the Bauschinger effect and the cross hardening.

- In addition, the previously formed dislocation walls are polarized. Here the term 'polarity' used to characterize the dislocation walls means that on each side of the dislocation wall there exists an excess of dislocations of the same sign, 


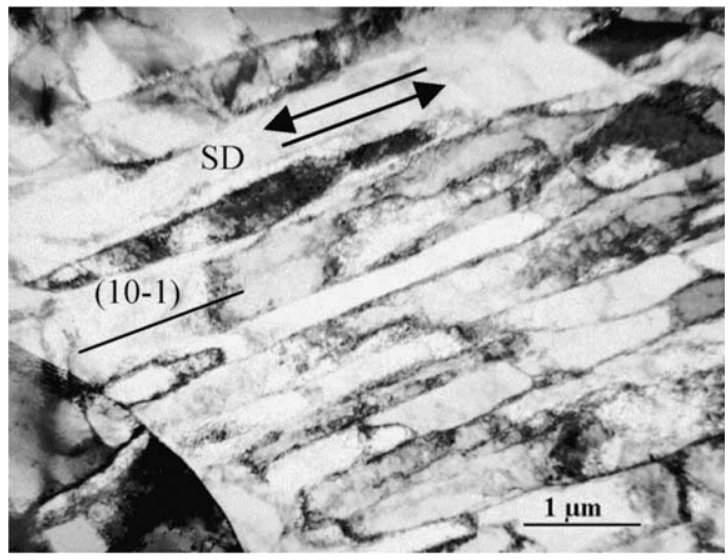

Fig. 1. Intragranular dislocation structures of $\gamma$-fibre grains after $60 \%$ amount of shear strain in an IF-mild steel from Nesterova et al. [12] (SD = shear direction).

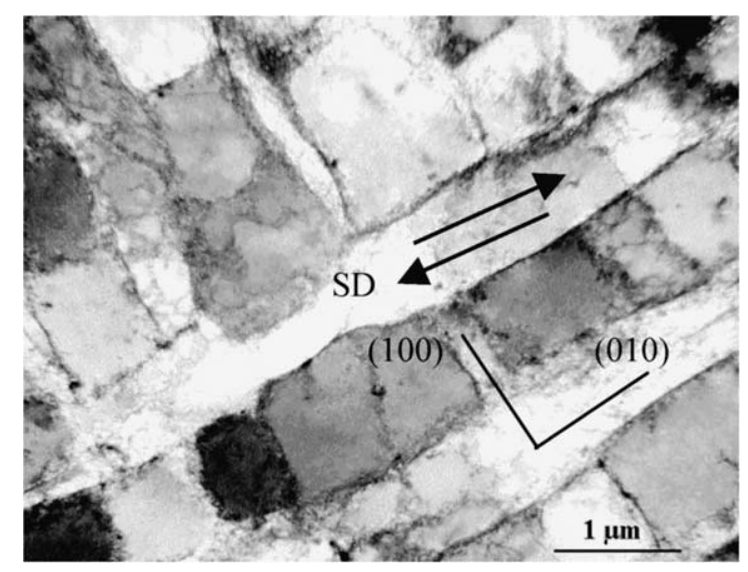

Fig. 2. Intragranular dislocation structures of a near cube grain after $15 \%$ amount of shear strain in an IF-mild steel from Nesterova et al. [13].

this sign being different on the opposite sides of the cell wall. This polarity attribute of the cell walls may be linked to the Bauschinger effect.

- In the case of quasi-monotonic strain-path change, TEM evidence shows that the hardening mechanisms is the same during the prestraining and the subsequent deformation and it consists of a continuous reinforcement of the wall structure. Therefore, no destabilization of the dislocation structure occurs, which leads to the absence of any transient behaviour on the macroscopic stress-strain curves.

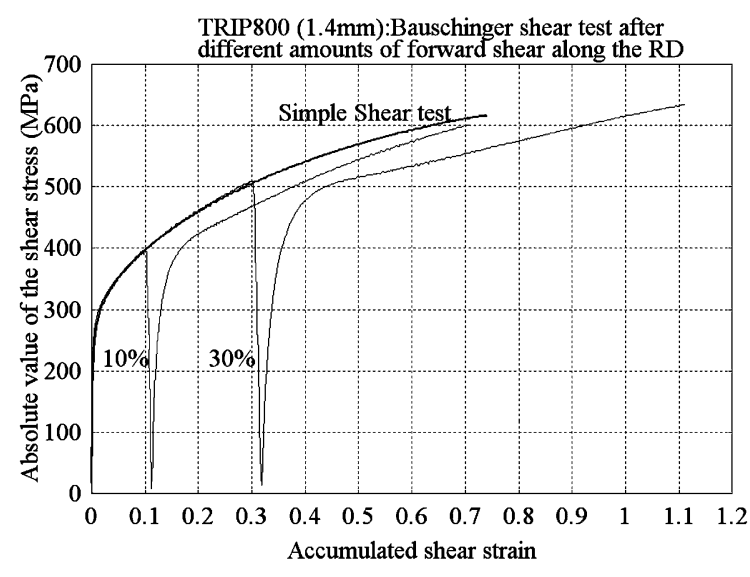

Fig. 3. Bauschinger strain-path change in a TRIP800 steel.

- Conversely, in the case of quasi-Bauschinger strain-path change (Fig. 3), the transient behaviour of the flow stress is correlated to the cell walls disintegration. In fact, since the load is reversed in most of the grains, it enables the dislocations to escape from the walls as the stress increases so that annihilation may take place. Therefore, the first stage of reversed deformation corresponds to the disturbance of preformed dislocation cell structures, the extended plateau in the stress-strain curve to the partial disintegration of the preformed structures, and the subsequent resumption of work-hardening to the formation of new cell structures of opposite polarity. The low yield stress observed is commonly explained as yielding assisted by the back stress of which microscopic counterpart is the internal stress due to dislocation pile-ups against microscopic barriers.

- In the case of an orthogonal strain-path change (Fig. 4), some slip systems activated during the previous deformation become latent and new slip systems are activated. The increase of the macroscopic flow stress at the beginning of the second stage of the deformation allow to conclude that the dislocation structures due to prestrain seems to act as obstacles to slip on the newly active systems. The macroscopic observed work-softening is obviously connected to the appearance of microbands which are marked by the dislocation walls formed 


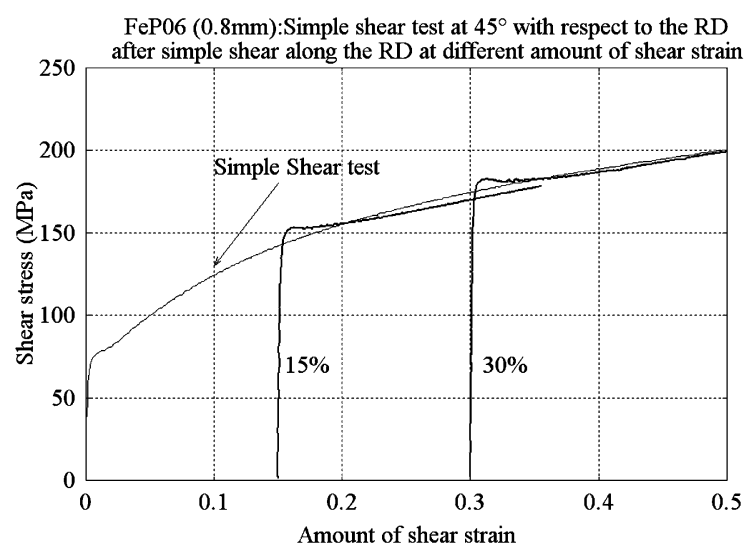

Fig. 4. Orthogonal strain-path change in a mild stell FeP06.

during the first stage of the deformation as depicted in Fig. 5: when microbands cross such walls, they create pronounced steps whose amplitude suggests that most of the deformation occurs in the microbands. Hence, strain localization appears as a mechanism related with a rapid change of the dislocation microstructure.

It is deduced from the TEM observations that the macroscopic stress reflects the local flow law connected to the formation and growth of microbands. A decrease of stress reveals that strong obstacles are broken down leading to microlocalized deformations. When the deformation increases on the second stage, further hardening process takes place due to the creation of newly dislocation walls.

\section{Modelling of the intragranular microstructure evolution}

Experimental investigations of two-stage strainpath tests reveal that the development of polarized dislocation sheets introduces a directional hardening of the material and that their polarity can explain the Bauschinger effect. In addition, the macroscopic work-hardening transients accompanying strain-path changes represent the phenomenological counterpart of the reorganization or dissolution of preformed dislocations structures and formation of new ones that correspond to the last deformation mode.

On the basis of the relationship between the flow-stress evolution and the microstructural evolution, Teodosiu and $\mathrm{Hu}[14,15]$ proposed a dislocation-based constitutive model, where the internal state variables are associated to microstructural components. The model is developed in the general framework of cold deformation of metals, neglecting any viscous effects on the work-hardening ${ }^{1}$. Only the contribution of the microstructural evolution and the influence of the initial texture are considered, since they are predominant at moderately large strains, whereas the influence of the texture evolution on the work-hardening is neglected.

After a brief recall of the classical setting of the rate-independent anisotropic plasticity, the dislocation-based microstructural model is described with its refinements subsequently introduced and the choice of the hardening laws involved is discussed. The microstructural model can be coupled with any of the recently published orthotropic and non-orthotropic yield criteria. All tensor variables are denoted by bold-face symbols, while their objective rates, which are considered of Jaumann type (co-rotational frame), are labelled by small superposed circles. We restrict in the following to the equations describing the plastic behaviour, referring for the coupling with hypoelastic constitutive equations within the framework of large elastoplastic deformations to the original paper of Teodosiu and $\mathrm{Hu}$ [14].

We assume that the yield condition has the form

$\Phi \equiv \bar{\sigma}-Y=0$,

where $Y$ is the yield stress, whose evolution describes the isotropic hardening. The equivalent effective stress is given by the general quadratic function

$\bar{\sigma}=\sqrt{\mathbf{s}: \mathbf{M}: \mathbf{s}}$,

\footnotetext{
${ }^{1}$ Recently, this model has been generalized to include rate and thermal effects, in particular during for high strain rate deformations (see [16]).
} 


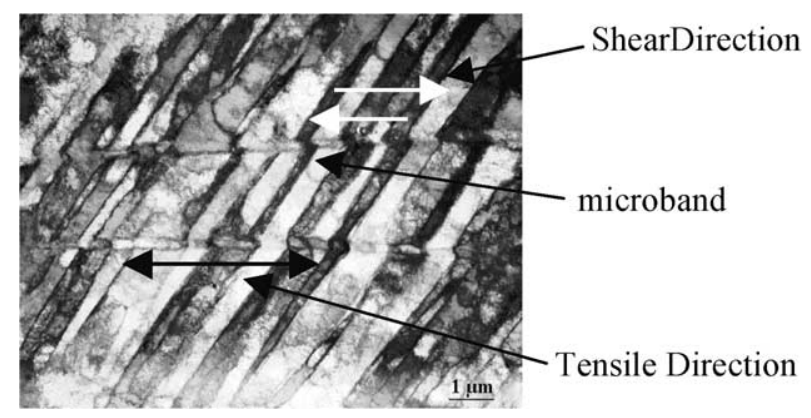

Fig. 5. TEM microstructure of ZStE180BH steel after 10\% tensile true strain in the RD followed by $10 \%$ amount of shear in the same direction from Nesterova et al. [10].

where $\mathbf{s}=\boldsymbol{\sigma}^{\prime}-\mathbf{X}$ is the effective stress tensor, $\boldsymbol{\sigma}^{\prime}$ is the deviator of the Cauchy stress tensor, $\mathbf{X}$ denotes the back-stress tensor, and $\mathbf{M}$ is a fourth-order tensor characterizing the texture anisotropy. We assume that the laminated sheet remains orthotropic during the deformation, whereas the orthotropy axes are subjected to a time-dependent rotation $\mathbf{R}$, whose evolution equation is $\dot{\mathbf{R}}=$ $\mathbf{W R}$, where $\mathbf{W}$ is the antisymmetric part of the velocity gradient. We take the effective stress $\bar{\sigma}$ in the form of Hill's quadratic function

$$
\begin{aligned}
\bar{\sigma}^{2}= & F\left(\hat{s}_{22}-\hat{s}_{33}\right)^{2}+G\left(\hat{s}_{33}-\hat{s}_{11}\right)^{2}+H\left(\hat{s}_{11}-\hat{s}_{22}\right)^{2} \\
& +2 L \hat{s}_{23}^{2}+2 M \hat{s}_{23}^{2}+2 N \hat{s}_{12}^{2}
\end{aligned}
$$

where $\hat{s}_{i j}$ are the components of the effective stress tensor in the axes of orthotropy.

The plastic strain rate, $\mathbf{D}^{\mathrm{p}}$, is given by the associated flow rule

$\mathbf{D}^{\mathrm{p}}=\dot{\lambda} \frac{\partial \Phi}{\partial \mathbf{s}}=\dot{\lambda} \frac{\partial \bar{\sigma}}{\partial \mathbf{s}}=\frac{\dot{\lambda}}{\bar{\sigma}} \mathbf{M}: \mathbf{s}$,

where $\dot{\lambda}$ is the plastic multiplier, the superposed dot indicates the time differentiation. The equivalent plastic strain rate $\dot{\bar{\varepsilon}}^{\mathrm{p}}$ is defined as the power conjugate of $\bar{\sigma}$, i.e.

$\bar{\sigma} \dot{\bar{\varepsilon}}^{\mathrm{p}}=\mathbf{s}: \mathbf{D}^{\mathrm{p}}$.

By introducing Eq. (5) into Eq. (6) and considering also the relation (3), it follows that $\dot{\lambda}=\dot{\bar{\varepsilon}}^{\mathrm{p}}$. The equivalent plastic strain is defined by

$\overline{\bar{\varepsilon}}^{\mathrm{p}}=\int_{0}^{t} \dot{\bar{\varepsilon}}^{\mathrm{p}} \mathrm{d} t=\int_{0}^{t} \frac{\mathbf{s}: \mathbf{D}^{\mathrm{p}}}{\bar{\sigma}} \mathrm{d} t$.

\subsection{Hardening models}

The set of the internal state variables used in the microstructural model to describe the rather complex behaviour of polycrystalline materials under strain-path changes and at large strains is denoted by $(R, \mathbf{X}, \mathbf{P}, \mathbf{S}) . R, \mathbf{X}$, and $\mathbf{S}$ have the dimension of stress, while $\mathbf{P}$ has no dimension. $\mathbf{S}$ is a fourthorder tensor, $\mathbf{X}$ and $\mathbf{P}$ are second-order tensors, and $R$ is a scalar. For well-annealed materials, the initial values of all these internal variables are set equal to zero. The scalar $R$ describes the isotropic work-hardening associated with the randomly distributed dislocations. The tensors $\mathbf{S}$ and $\mathbf{P}$ are associated, respectively, with the directional strength of planar dislocation sheets and with their polarity. Finally, the tensor $\mathbf{X}$, called the backstress, is intended to describe the rapid changes in the flow stress following strain-path changes.

The yield condition is written in the form

$\Phi \equiv \bar{\sigma}-Y_{0}-R-f\|\mathbf{S}\|=0$,

where the terms $R$ and $f\|\mathbf{S}\|$ describe the contributions of the randomly distributed dislocations and of the organized dislocation structures to the isotropic hardening, respectively. $Y_{0}$ is the initial yield stress. The definition of the equivalent effective stress and the associated flow rule preserve the forms (3) and (5), respectively.

The evolution law for $\mathbf{P}$ is postulated in the form:

$\stackrel{\circ}{\mathbf{P}}=C_{\mathrm{P}}(\mathbf{A}-\mathbf{P}) \dot{\lambda}, \quad \mathbf{P}(0)=\mathbf{0}$, 
where $\mathbf{A}=\mathbf{D}^{\mathrm{p}} /\left\|\mathbf{D}^{\mathrm{p}}\right\|$ is the direction of the strain rate tensor and $C_{\mathrm{P}}$ is a material parameter defining the rate of evolution of the tensor $\mathbf{P}$. The evolution equation of the internal state variable $R$ is postulated in the form:

$\dot{R}=C_{\mathrm{R}}\left(R_{\mathrm{sat}}-R\right) \dot{\lambda} \quad$ and $\quad R(0)=0$,

where $C_{\mathrm{R}}$ and $R_{\text {sat }}$ are material parameters. The evolution law proposed for $R$ is the classical Voce law used for materials that exhibit a saturated isotropic hardening under monotonic loading. An another common evolution law that can be used to describe the evolution of the internal variable $R$ is the Swift law defined by

$R=C\left(\varepsilon_{0}+\bar{\varepsilon}^{\mathrm{p}}\right)^{n}$.

where $\varepsilon_{0}, C, n$ are material parameters. The initial value of the yield stress in this case is given by the relation $Y_{0}=C \varepsilon_{0}^{n}$ and the associated yield condition is given by $\Phi \equiv \bar{\sigma}-R-f\|\mathbf{S}\|=0$. This evolution law is adequate for describing the behaviour of materials that exhibit a non-saturated isotropic hardening.

The evolution equation of the back stress $\mathbf{X}$, is governed by the equations

$\stackrel{\circ}{\mathbf{X}}=C_{X}\left[\frac{X_{\text {sat }}}{\bar{\sigma}}\left(\boldsymbol{\sigma}^{\prime}-\mathbf{X}\right)-\mathbf{X}\right] \dot{\lambda}, \quad \mathbf{X}(0)=\mathbf{0}$,

where $X_{\text {sat }}$ characterizes the saturation value of the kinematic hardening, while the material parameter $C_{X}$ characterizes its rate of approaching the saturation. In case of plastic isotropy, this equation, generally attributed to Armstrong and Frederick [17], coincides with the saturation law which has been thoroughly investigated by Lemaitre and Chaboche [18]:

$\stackrel{\circ}{\mathbf{X}}=K \mathbf{D}^{\mathrm{p}}-\gamma \mathbf{X} \dot{\lambda}, \quad \mathbf{X}(0)=\mathbf{0}$,

when setting $K=(2 / 3) C_{X} X_{\text {sat }}$ and $\gamma=C_{X}$ The material parameters in Eq. (13) are $K$ and $\gamma$, where the ratio $K / \gamma$ characterizes the saturation value of the kinematic hardening, while $\gamma$ characterizes its rate of approaching the saturation. However, if the material is plastically anisotropic, Eq. (12) imply that, when no rotation takes place and $\boldsymbol{\sigma}^{\prime}$ is constant, the back stress tends to become coaxial with the deviatoric Cauchy stress at large values of the equivalent plastic strain, whereas Eq. (13) predicts that the back stress becomes coaxial with the plastic strain rate tensor, provided that the latter is maintained constant. The present authors prefer the former assumption, because it corresponds to the very definition of the back stress as opposing the applied shear stress on each slip system.

The saturation value $X_{\text {sat }}$ in the Eq. (12) is no longer constant, but it evolves with the persistent dislocation structures via the internal state variable $\mathbf{S}$, following the form

$X_{\text {sat }}=X_{0}+(1-f) \sqrt{r\|\mathbf{S}\|^{2}+(1-r) S_{\mathrm{D}}^{2}}$,

where $X_{0}$ is the initial value of $X_{\text {sat }}, r$ is a material parameter, and $S_{\mathrm{D}}=\mathbf{A}: \mathbf{S}: \mathbf{A}$ is the strength of the dislocation structures associated with the currently active slip systems, while $\mathbf{S}_{\mathrm{L}}$ is the part of $\mathbf{S}$ associated with the latent slip systems, and is defined by $\mathbf{S}_{\mathrm{L}}=\mathbf{S}-S_{\mathrm{D}} \mathbf{A} \otimes \mathbf{A}$. By introducing the parameter $\beta_{\mathrm{S}}=S_{\mathrm{D}} /\|\mathbf{S}\|$ proposed by Teodosiu and $\mathrm{Hu}$ [1], Eq. (14) becomes

$X_{\mathrm{sat}}=X_{0}+(1-f)\|\mathbf{S}\| \sqrt{r+(1-r) \beta_{\mathrm{S}}^{2}}$.

$\beta_{\mathrm{S}}$ parameter evolves from 0 to 1 for the different strain-path change. Hence, when the material parameter $r>1, X_{\text {sat }}$ has the highest value for $\beta_{\mathrm{S}}=0$ (orthogonal loading) and the lowest one for $\beta_{\mathrm{S}}=1$ (monotonic or Bauschinger loading). This parameter characterizes the interaction between previously formed persistent dislocation structures and the current strain rate mode. Regarding to microstructural evidence, the evolution of $\mathbf{X}$ should be more rapid than that of $\mathbf{S}$. This is characterized by $C_{X}$ which has to be greater than $C_{\mathrm{SD}}$ (in Eq. (16)). The large hardening rate at the beginning of the second path corresponds to this evolution. The following decrease in the rate of hardening is associated with the saturation of $\mathbf{X}$ towards $X_{\text {sat }}$ (Figs. 6 and 7).

Following TEM observations in ferritic steels, two physical mechanisms are possible immediately after an orthogonal strain-path change: the partial disintegration of the preformed dislocation structures or the softening of the preformed dislocation structures after being sheared by newly generated 


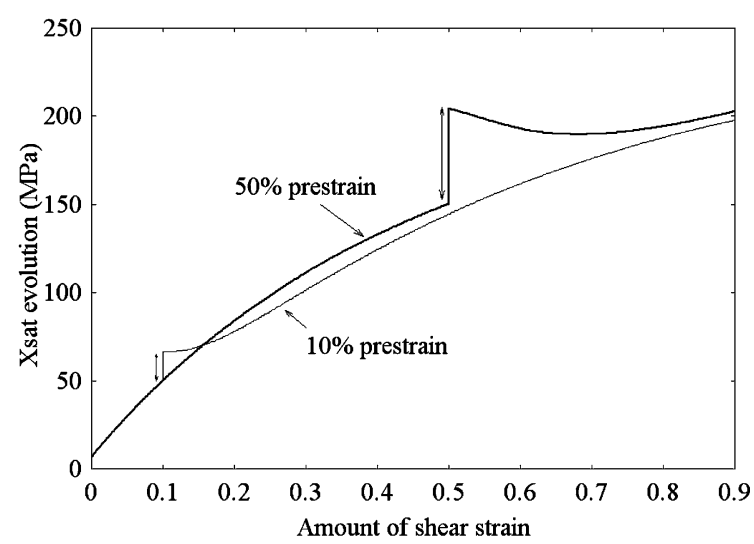

Fig. 6. $X_{\text {sat }}$ evolution after orthogonal strain-path change.

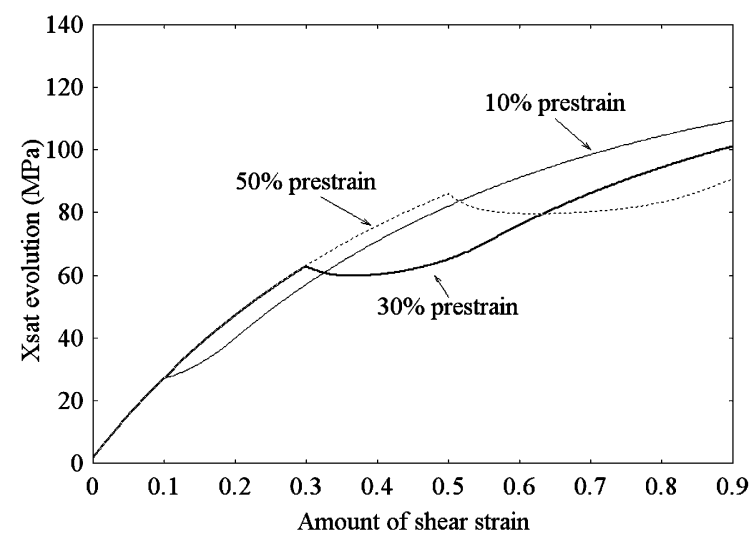

Fig. 7. $X_{\text {sat }}$ evolution after pseudo-Bauschinger strain-path change.

microbands. Both these mechanisms reduce the intensity of the latent part $\mathbf{S}_{\mathrm{L}}$ of the dislocation structures, whereas the part $S_{\mathrm{D}}$ associated with the currently active slip systems will increase. Hence, the following evolution equations of $S_{\mathrm{D}}$ and $\mathbf{S}_{\mathrm{L}}$ are adopted:

$$
\left\{\begin{array}{l}
\stackrel{\circ}{\mathrm{S}}_{\mathrm{L}}=-C_{\mathrm{SL}}\left(\frac{\left\|\mathbf{S}_{\mathrm{L}}\right\|}{S_{\mathrm{sat}}}\right)^{n_{\mathrm{L}}} \mathbf{S}_{\mathrm{L}} \dot{\lambda} \\
\dot{S}_{\mathrm{D}}=C_{\mathrm{SD}}\left[g\left(S_{\mathrm{sat}}-S_{\mathrm{D}}\right)-h S_{\mathrm{D}}\right] \dot{\lambda}
\end{array}\right.
$$

where $C_{\mathrm{SD}}$ and $C_{\mathrm{SL}}$ characterize the saturation rate of $S_{\mathrm{D}}$ and $\mathrm{S}_{\mathrm{L}}$, respectively, $S_{\mathrm{sat}}$ denotes the saturation value of $S_{\mathrm{D}}$. For well-annealed materials, the initial value of $\mathbf{S}$ equals $\mathbf{0}$, and hence also $S_{\mathrm{D}}$ and $\mathrm{S}_{\mathrm{L}}$ have zero initial values. As long as the loading remains monotonic, $\mathbf{S}_{\mathrm{L}}$ remains equal to $\mathbf{0}$. In order to model the stagnation and resumption of the work-hardening observed after Bauschinger strain-path changes, the scalar function $g$ is introduced. It describes the influence of the polarity of the persistent dislocation structures on the evolution of the work-hardening. The function $g$ depends on the projection $P_{\mathrm{D}}=\mathbf{P}: \mathbf{A}$ of the polarity tensor $\mathbf{P}$ on the current direction of the strain rate A. Explicitly,

$g= \begin{cases}1-\frac{C_{\mathrm{P}}}{C_{\mathrm{SD}}+C_{\mathrm{P}}}\left|\frac{S_{\mathrm{D}}}{S_{\mathrm{sat}}}-P_{\mathrm{D}}\right| & \text { if } P_{\mathrm{D}} \geqslant 0, \\ \left(1+P_{\mathrm{D}}\right)^{n_{\mathrm{P}}}\left(1-\frac{C_{\mathrm{P}}}{C_{\mathrm{SD}}+C_{\mathrm{P}}} \frac{S_{\mathrm{D}}}{S_{\mathrm{sat}}}\right) & \text { otherwise }\end{cases}$

where $n_{\mathrm{P}}$ is a positive material parameter. Regarding to the evolution laws proposed for $g$, when the material is first severely deformed at a constant strain rate direction $\mathbf{A}_{1}$, the polarity tensor $\mathbf{P}$ end up being practically equal to $\mathbf{A}_{1}$ (see Eq. (9)) and $P_{\mathrm{D}}=1$ and $g$ decreases gradually from 1 . In addition, $S_{\mathrm{D}}$ tends to be equal to $S_{\text {sat }}$ (see Eq. (16)). If the material is subsequently subjected to a reversed deformation, i.e. $\mathbf{A}=-\mathbf{A}_{1}$, then $P_{\mathrm{D}}$ takes abruptly the value -1 and $g$ the value 0 . According to the evolution law of $S_{\mathrm{D}}\left(S_{\mathrm{D}}=S_{\mathrm{sat}}\right.$ at the end of the first strain-path), the last condition corresponds to the stagnation of the work-hardening. As the strain increases along the reverse direction, $P_{D}$ increases gradually towards positive values, leading to the increase of $g$ (Fig. 8) and to the resumption of the work-hardening.

$h$ is a scalar function defined as

$h=\frac{1}{2}\left(1-\frac{\mathbf{X}: \mathbf{A}}{X_{\mathrm{sat}}}\right)$,

It describes the slight variation of $S_{\mathrm{D}}$ at the beginning of the reversed deformation in a Bauschinger test (Fig. 9) and has a non-negligible value only during a microplastic stage.

Finally, in order to illustrate how the physicsbased microstructural model allows to reproduce the non-isotropic behaviour due to the microstructural evolution, the relationship between the hardening behaviour and the evolution of the internal state variables are succinctly investigated. 


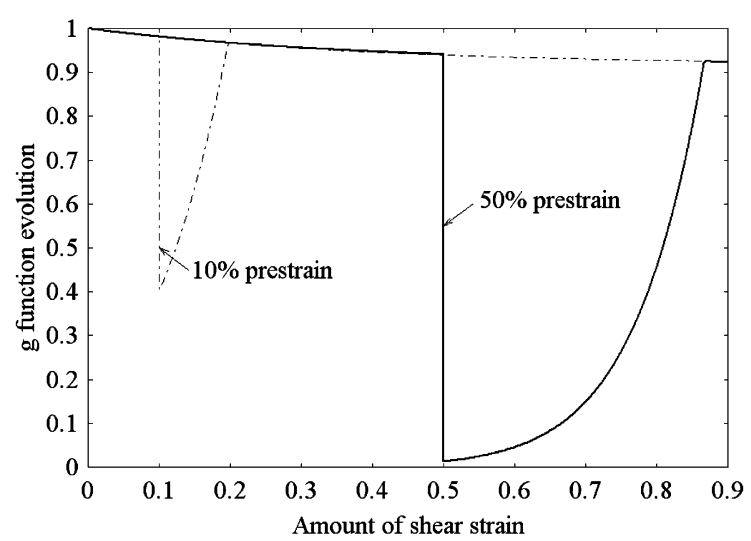

Fig. 8. Evolution of the scalar function $g$ during the quasiBauschinger strain-path loading.

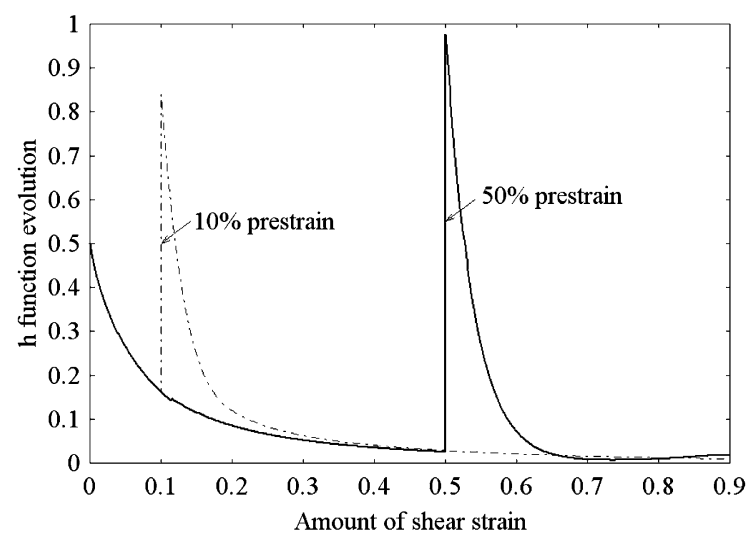

Fig. 9. Evolution of the scalar function h during the quasiBauschinger strain-path loading.

Figs. 10 and 11 give the work-hardening rates evolution after simulations of an orthogonal strain-path change and a quasi-Bauschinger strainpath change. The minimum of the rate of workhardening observed is due to the evolution of $\mathbf{S}$ and $X_{\text {sat }}$. Indeed, at the beginning of the second straining, the increasing rates of $\mathbf{S}$ and $X_{\text {sat }}$ are slower than those before the strain-path change, or even negative for a large prestrain (Figs. 6, 7, 12 and 13). As the strain increases along the second strain-path, the evolution rates of $\mathbf{S}$ and $X_{\text {sat }}$ increase gradually and the associated curves are concave downwards to upwards. This is the reason why a minimum is observed in the evolution of the rate of work-hardening.

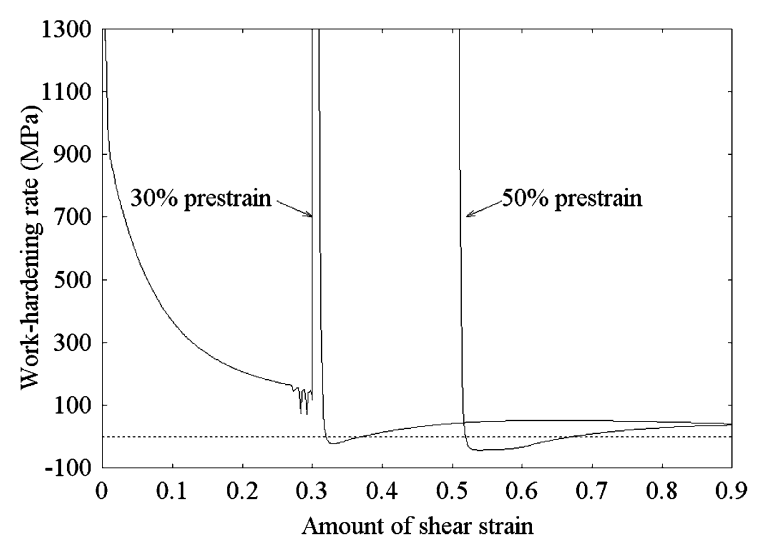

Fig. 10. Work-hardening rate evolution after the simulation of an orthogonal strain-path change.

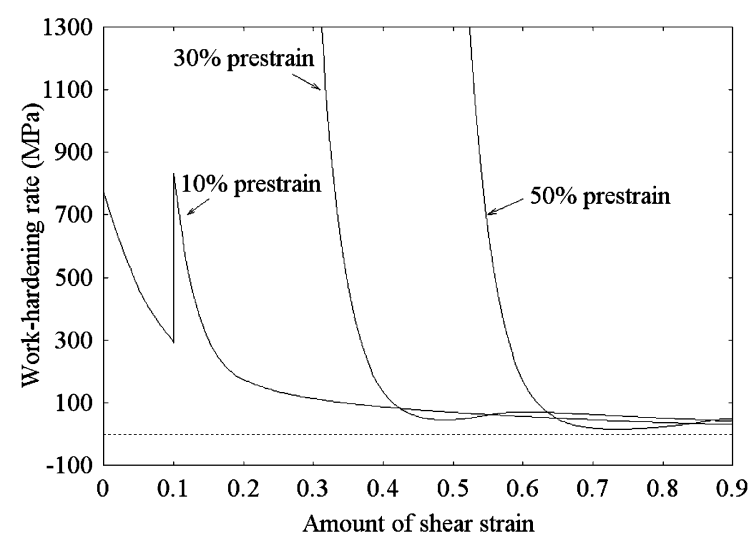

Fig. 11. Work-hardening rate evolution after a simulation of a quasi-Bauschinger loading.

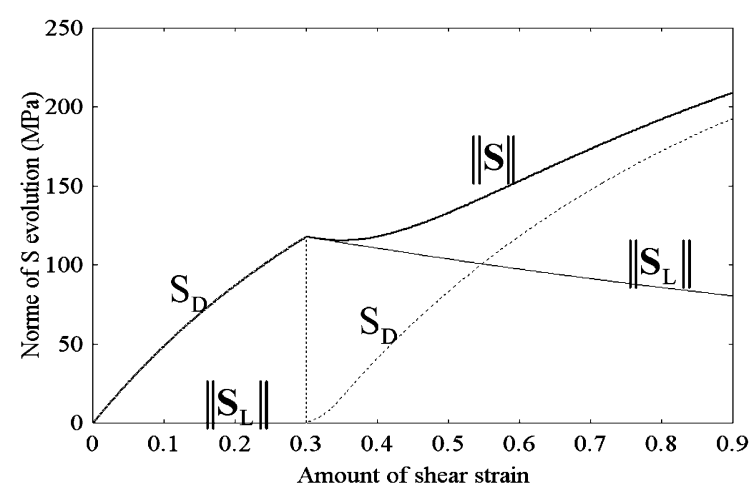

Fig. 12. $\|\mathbf{S}\|$ evolution after orthogonal strain-path change. 


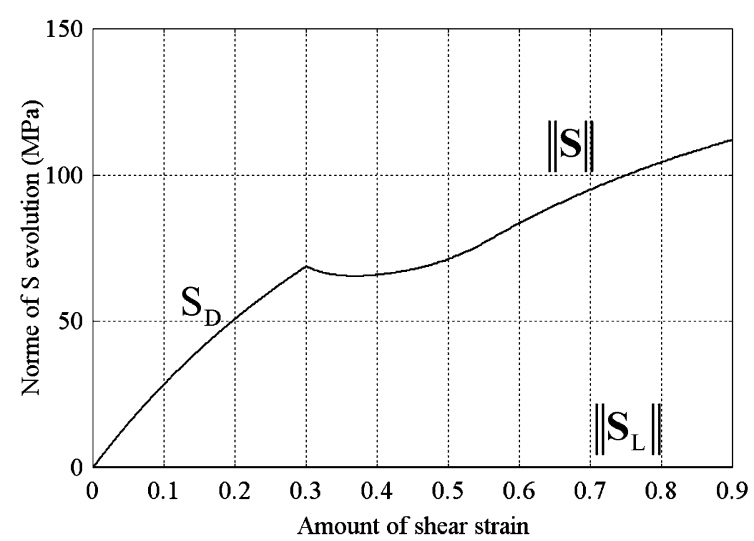

Fig. 13. $\|\mathbf{S}\|$ evolution after pseudo-Bauschinger strain-path change.

This evolution of $\mathbf{S}$ and $X_{\text {sat }}$ results from the evolution of $\mathbf{S}_{\mathrm{L}}$ which appears when the strainpath is changed (excluding the Bauschinger loading, Fig. 13). The value of $\mathbf{S}_{\mathrm{L}}$ at the beginning of the second strain increases with the amount of prestrain. As the second strain goes on, $\mathbf{S}_{\mathrm{L}}$ will decrease (Fig. 12). According to Teodosiu and $\mathrm{Hu}$ [1], the evolution of $S_{\mathrm{L}}$ is attributed to the interaction between microbands and the previously developed microstructures, and it was modelled in such a way that $\mathbf{S}_{\mathrm{L}}$ decreases more rapidly as the prestrain increases and, hence, the minimum of the rate of work-hardening decreases with the increase in prestrain.

Regarding the definition of $S_{\mathrm{D}}$ and $\mathrm{S}_{\mathrm{L}}$, the norm of $\mathbf{S}$ is equal to $\sqrt{S_{\mathrm{D}}^{2}+\left\|\mathbf{S}_{\mathrm{L}}\right\|^{2}}$. Hence, the evolution law of $X_{\text {sat }}$ can be rewritten as

$X_{\text {sat }}=X_{0}+(1-f) \sqrt{r\left\|\mathbf{S}_{\mathrm{L}}\right\|^{2}+S_{\mathrm{D}}^{2}}$.

Assuming that the material parameter $r$ is higher than 1, means that the latent dislocations (described by $S_{\mathrm{L}}$ ) have higher effectiveness as a source of the back stress than the currently active dislocations (described by $S_{\mathrm{D}}$ ). Therefore, the hardening stagnation or the softening are more pronounced in $X_{\text {sat }}$ than in S. Afterwards, the rate of workhardening starts increasing, because the evolution rate of $S_{\mathrm{D}}$ is higher than that of $S_{\mathrm{L}}$. As the second deformation goes on, $\mathbf{S}_{\mathrm{L}}$ and $S_{\mathrm{D}}$ saturate towards 0 and $S_{\text {sat }}$, respectively. This corresponds to the saturation of the persistent dislocation structures towards that obtained after large monotonic straining. As a result, the evolution rates of $\mathbf{S}$ and $X_{\text {sat }}$ decrease again.

In its extensive form, which is used for mild steels, the microstructural model involves 13 material parameters, namely $Y_{0}, C_{\mathrm{R}}, R_{\mathrm{sat}}\left(\right.$ or $\left.C, \varepsilon_{0}, n\right), f$, $C_{X}, X_{0}, C_{\mathrm{P}}, C_{\mathrm{SD}}, S_{\mathrm{sat}}, n_{\mathrm{P}}, C_{\mathrm{SL}}, r, n_{\mathrm{L}}$. These parameters may be identified, e.g., by using: (i) a uniaxial tensile test along the rolling direction up to localized necking, (ii) a monotonic simple shear test along the rolling direction up to $80 \%$ amount of shear, (iii) three Bauschinger simple shear tests along the rolling direction, after 10\%, 20\% and $30 \%$ amount of forward shear, and (iv) an orthogonal strain-path change, realized by first imposing a true tensile strain of $20 \%$ in the rolling direction, and then shearing in the same direction.

The microstructural model can be reduced to more classical phenomenological models by simply imposing zero value to a reduced set of material parameters as indicated in Table 1.

\section{Numerical implementation in DD3IMP finite element code}

The microstructural model presented above was implemented in the in-house finite element code DD3IMP (Deep Drawing 3D IMPlicit Code). The evolution of the deformation process is described by using an updated Lagrangian scheme. Large elastoplastic strains and rotations are taken into account and it is assumed that the elastic strains are negligibly small with respect to unit and that the elastic behaviour is isotropic. The plastic behaviour of the material is described by several orthotropic yield criteria. In the present work, the classical quadratic Hill'48 yield criterion is adopted.

An explicit approach is used to calculate an approximate first solution for incremental displacements, stresses and frictional contact forces. An $r_{\text {min }}$ strategy is employed to impose limitations on the size of the time increments. This first solution satisfies neither the variational principle nor the coherence condition, due to the nonlinearities of the problem. Subsequently, this first approximation of the configuration and of the state of 
Table 1

Classification of the elastoplastic models

\begin{tabular}{lll}
\hline Models & Material parameters & Material parameters equal to 0 \\
\hline Isotropic hardening (Swift law) & $C, \varepsilon_{0}, n$, & $f, C_{X}, X_{0}, C_{\mathrm{P}}, C_{\mathrm{SD}}, S_{\mathrm{sat}}, n_{\mathrm{P}}$, \\
& & $C_{\mathrm{SL}}, r, n_{\mathrm{L}}$ \\
& & $f, C_{\mathrm{P}}, C_{\mathrm{SD}}, S_{\mathrm{sat}}, n_{\mathrm{P}}, C_{\mathrm{SL}}, r, n_{\mathrm{L}}$ \\
Isotropic hardening (Swift law) + kinematic hardening & $C, \varepsilon_{0}, n, C_{X}, X_{0}$ & $f, C_{X}, X_{0}, C_{\mathrm{P}}, C_{\mathrm{SD}}, S_{\mathrm{sat}}, n_{\mathrm{P}}$, \\
Isotropic hardening (Voce law) & $Y_{0}, C_{\mathrm{R}}, R_{\mathrm{sat}}$, & $C_{\mathrm{SL}}, r, n_{\mathrm{L}}$ \\
& & $f, C_{\mathrm{P}}, C_{\mathrm{SD}}, S_{\mathrm{sat}}, n_{\mathrm{P}}, C_{\mathrm{SL}}, r, n_{\mathrm{L}}$ \\
Isotropic hardening (Voce law) + kinematic hardening & $Y_{0}, C_{\mathrm{R}}, R_{\mathrm{sat}}, C_{X}, X_{0}$ & \\
Complete microstructural model & $Y_{0}, C_{\mathrm{R}}, R_{\mathrm{sat}}\left(\mathrm{or} C, \varepsilon_{0}, n\right), f$, & \\
& $C_{X}, X_{0}, C_{\mathrm{P}}, C_{\mathrm{SD}}, S_{\mathrm{sat}}, n_{\mathrm{P}}, C_{\mathrm{SL}}, r, n_{\mathrm{L}}$ \\
\hline
\end{tabular}

the deformed body at instant $t+\Delta t$, is successively corrected using an implicit method, until the equilibrium of the deformable body is satisfactory. It is now possible to update the configuration of the sheet at the end of the increment $[t, t+\Delta t]$ and all the state variables, passing on therefore to the next increment calculation until the end of the process [19]. The global algorithm of DD3IMP is summarized in Table 2.

The geometry of the forming tool is modelled by parametric surfaces (Bézier surfaces); continuity $C^{0}$ and $C^{1}$ should be guaranteed for best results. The Coulomb classical law models the frictional contact problem between a deformable body (the blank) and a rigid body (the tool), which is treated with an augmented Lagrangian approach [19]. A fully implicit algorithm of Newton-Raphson type

Table 2

Global algorithm of DD3IMP code

Input and check data
Repeat
Impose a trial increment
Calculate the explicit tangent stiffness matrix
Solve system for the trial increments displacements
Choose the trial increments by a $r_{\text {min }}$ strategy
Update sheet configuration and tools positions
Change contact/uncontact boundary conditions
Repeat ! Using an implicit algorithm
Calculate incremental strains and rotation
Integrate the constitutive law
Calculate residual forces
Solve system for the incremental displacements
Update sheet configuration, state variables and adjust
boundary conditions
Until residual non-equilibrated forces are close to zero
Until the end of the process
Output results

is used to solve the non-linearities related with the frictional contact problem and the elastoplastic behaviour of the deformable body[20]. The blank is discretized with 3D solid finite elements [21]. This allows an accurate calculation of the stress gradients through thickness of the sheet as well as the thickness evolution. For more details of the numerical implementation of the microstructural model, the present authors refer to their recently published papers $[19,21,22]$.

\section{Numerical example}

The deep drawing problem selected for the analysis of the influence of the work hardening constitutive models is a curved rail developed under the 3DS project [23]. This rail was specially conceived to emphasize 3D springback effects. The main dimensions of the forming tool (die, blank holder and punch) are presented in Fig. 14. The deep drawing process is divided in three phases. First the blank holder is moved until it reaches the initial prescribed force. Then the punch starts its movement up to a total displacement of $60 \mathrm{~mm}$. During this phase the blank holder force increases proportionally to the punch displacement to a maximum value of $118 \mathrm{kN}$. The final phase is the springback simulation (removal of the tool).

The blank sheet is a square with $300 \mathrm{~mm}$ length and $1.0 \mathrm{~mm}$ thickness. Due to the geometrical and material symmetries only a half of the structure was considered in the simulations. Following previous works, the blank is discretized with a nonuniform mesh of eight-node hexahedron solid finite elements combined with a selective reduced 


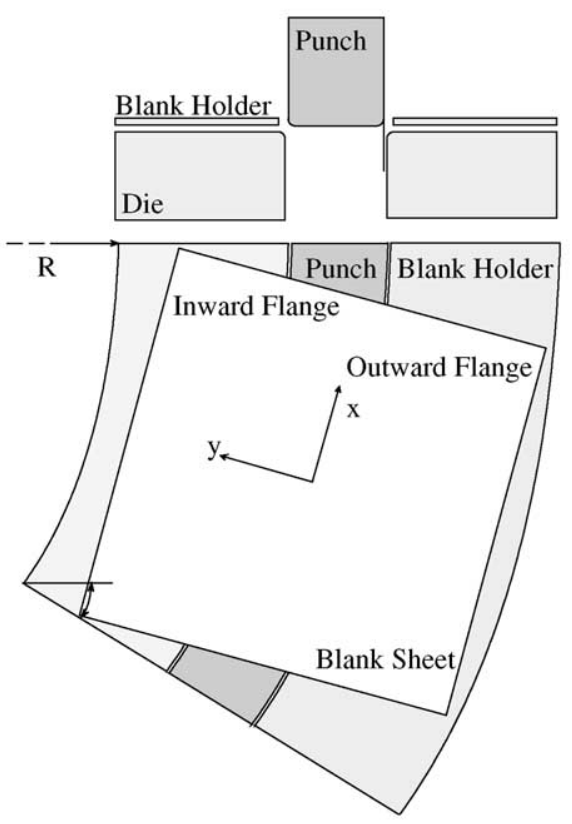

(a)

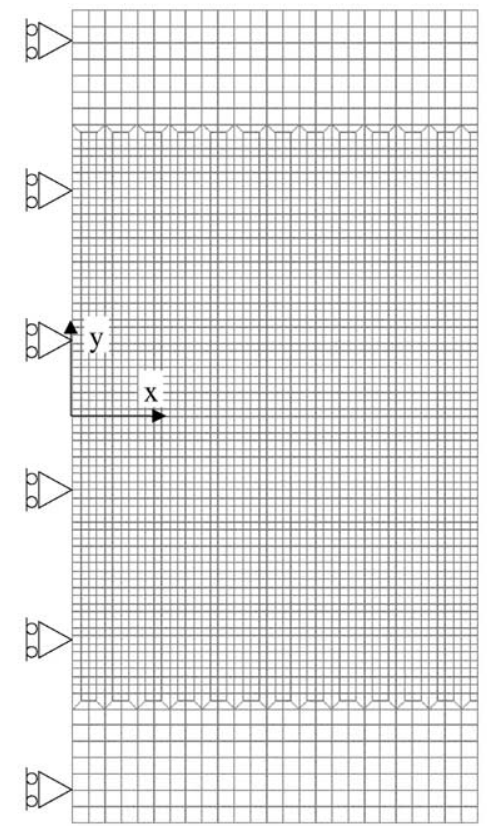

(b)

Fig. 14. (a) Forming tool for the deep drawing of the curved rail and (b) the finite element mesh.

integration technique [21]. The average element size on the flat contact zone between the blank holder and the die has $6.0 \mathrm{~mm}$ and it is reduced to $3.0 \mathrm{~mm}$ in the other contact areas. Only one layer of elements is used in thickness, although previous works indicate that this may affect the accuracy of the results [22].

The results obtained with a more refined phenomenological model are compared with the ones obtained from some classical constitutive models, namely two isotropic hardening models, combined or not with a non-linear kinematic hardening. It is worth noting that, except for the microstructural model, the material parameters used in the actual simulations have been identified by best fit of the experimental values and giving the same weight to all mechanical tests. In case of the microstructural model, however, since some of its parameters describe specific parts of the stress-strain curves the weights given to such parts were in general different.

The material used for the simulations is a high strength low alloyed steel HSLA340. The values of its hardening parameters are [24]: $Y_{0}=367.7 \mathrm{MPa}, \quad C_{\mathrm{R}}=32.2, \quad R_{\mathrm{sat}}=23.6 \mathrm{MPa}$,
$C_{X}=36.1, X_{0}=54.2 \mathrm{MPa}, C_{\mathrm{SD}}=5.72, C_{\mathrm{SL}}=0$, $S_{\text {sat }}=137.9 \mathrm{MPa}, n=0, n_{\mathrm{P}}=290, r=0.58, f=0$, $C_{\mathrm{P}}=0.442$ (this model is referred ahead to $\mathrm{MM}$ ). In order to investigate the effect of the material law on the finite element results, more classical phenomenological laws are identified, namely: Swift law or Voce law combined with a non-linear kinematic hardening. The related material parameters are given in Table 3.

Finally, the anisotropy parameters for the Hill'48 criterion are identified using a best fit of the evolution curve of the experimental value of the Hill coefficient of anisotropy $r(\alpha): F=0.414$, $G=0.558, H=0.442, L=M=1.5$ and $N=1.52$.

\subsection{Results and discussion}

The punch force evolution, obtained with the five different work hardening constitutive models is presented in Fig. 15. These results allow to conclude that the punch force evolution is in general of the same magnitude for all work hardening constitutive models. In order to quantify the differences induced by the mechanical characterization, the 
Table 3

Material parameters of the HSLA

\begin{tabular}{|c|c|c|c|c|c|c|c|}
\hline \multicolumn{2}{|c|}{ Swift law } & \multicolumn{2}{|c|}{$\begin{array}{l}\text { Swift law }+ \text { KH } \\
\text { (w/kinetic hardening) }\end{array}$} & \multicolumn{2}{|c|}{ Voce law } & \multicolumn{2}{|c|}{$\begin{array}{l}\text { Voce law + KH } \\
\text { (w/kinetic hardening) }\end{array}$} \\
\hline$Y_{0}$ & 365.3 & $Y_{0}$ & 367.7 & $Y_{0}$ & 365.3 & $Y_{0}$ & 367.7 \\
\hline$n$ & 0.131 & $n$ & 0.139 & $C_{\mathrm{R}}$ & 7.5 & $C_{\mathrm{R}}$ & 3.64 \\
\hline \multirow[t]{3}{*}{$C$} & 673.0 & $C$ & 530.9 & $R_{\text {sat }}$ & 247.8 & $R_{\text {sat }}$ & 147.1 \\
\hline & & $C_{X}$ & 20.7 & & & $C_{X}$ & 24.7 \\
\hline & & $X_{\text {sat }}$ & 98.0 & & & $X_{\text {sat }}$ & 95.4 \\
\hline
\end{tabular}

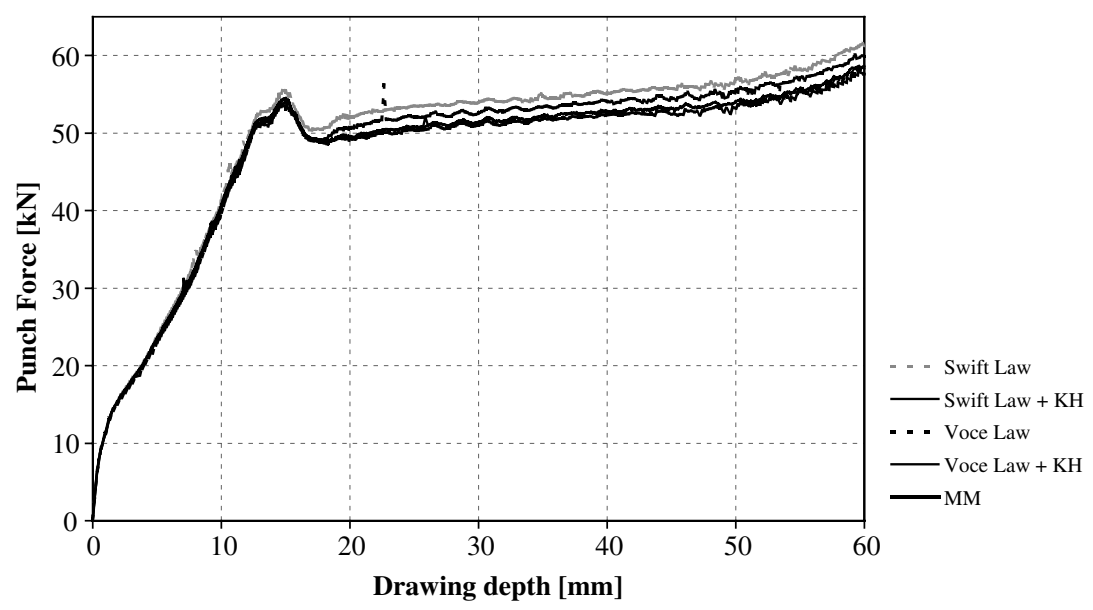

Fig. 15. Variation of the punch force with the punch displacement during the deep drawing of the curved rail.

isotropic work hardening described by the Swift law without kinematic hardening is defined as reference. This choice reflects the traditional options available in the majority of the deep-drawing simulation codes. The introduction of the kinematic work hardening in both isotropic hardening models (Swift and Voce laws) leads to a reduction of the maximum punch force of around 5\%, when compared to the results obtained without kinematic hardening. The reduction obtained with the microstructural model is of around $6 \%$.

In order to evaluate the differences in terms of the final geometry, the draw-in of the inward an outward flange (see Fig. 14) is presented in Fig. 16. Due to the shape of this curved rail, the draw-in of the inward flange is always higher than the draw-in of the outward flange. Globally the final shapes of the flanges are in good agreement with the expected For this parameter only, the test performed with the isotropic hardening described by the Swift law leads to clearly different results.

Two transversal sections were selected to evaluate springback differences. Section 1 corresponds to a zone close to rail edge, and Section 2 corresponds to the symmetry plane. The results obtained with the different behaviour laws are presented in Figs. 17 and 18, for Sections 1 and 2 , respectively.

The springback angles were evaluated with the software "NXT Post Processor II" ( ${ }^{2}$ Fig. 19 shows the springback angle predicted for both the inward and outward flange, in both sections. The springback of the inward flange seems to be more sensible to the material behaviour then the springback of the outward side of the part. Also,

\footnotetext{
${ }^{2}$ M\&M Research, Inc. http://www.m-research.co.jp, support@m-research.co.jp.
} 


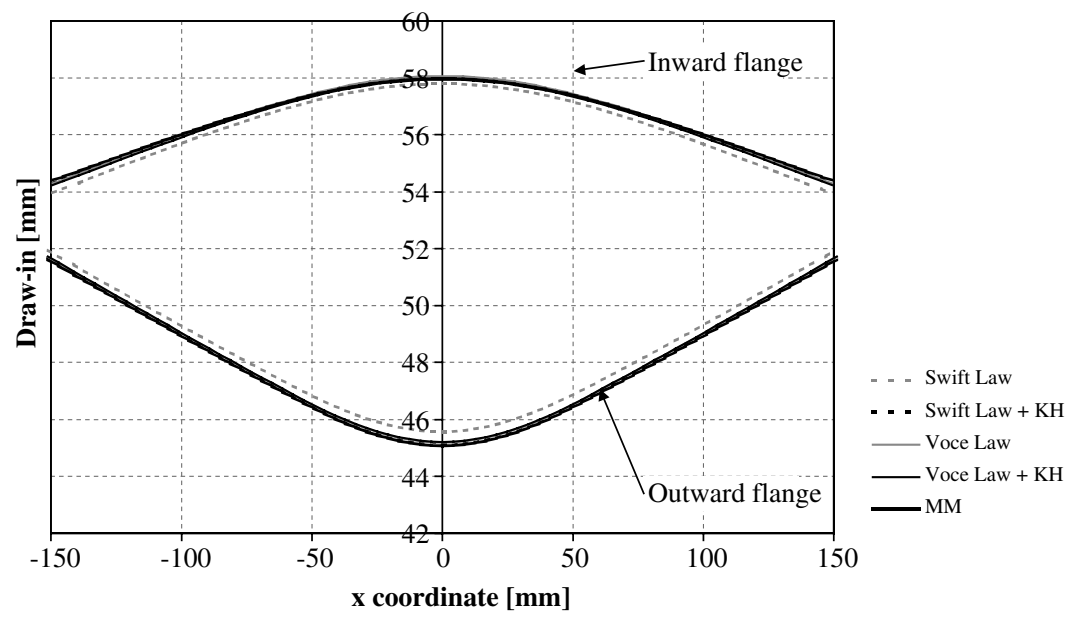

Fig. 16. Shapes of inward and outward flanges after springback.

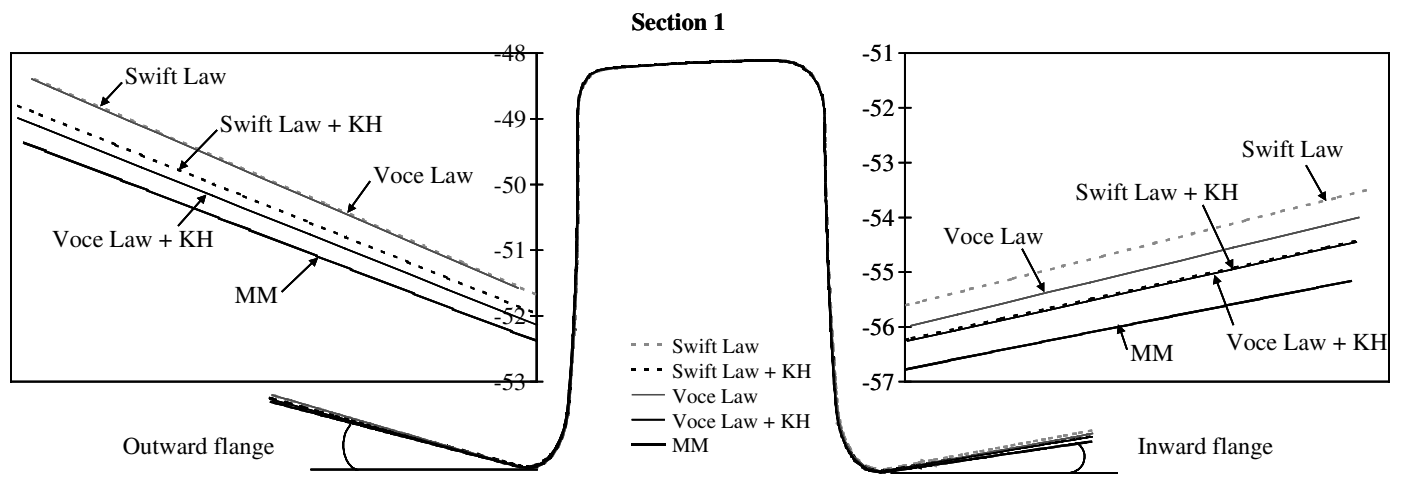

Fig. 17. Profile of Section 1 predicted after springback.

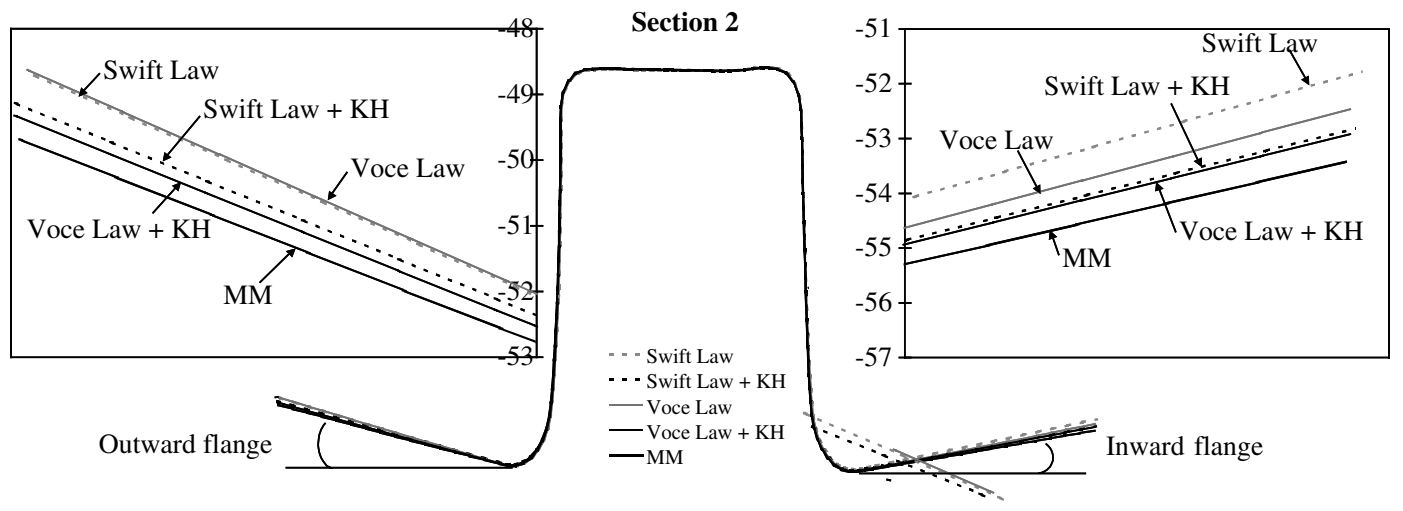

Fig. 18. Profile of Section 2 predicted after springback. 


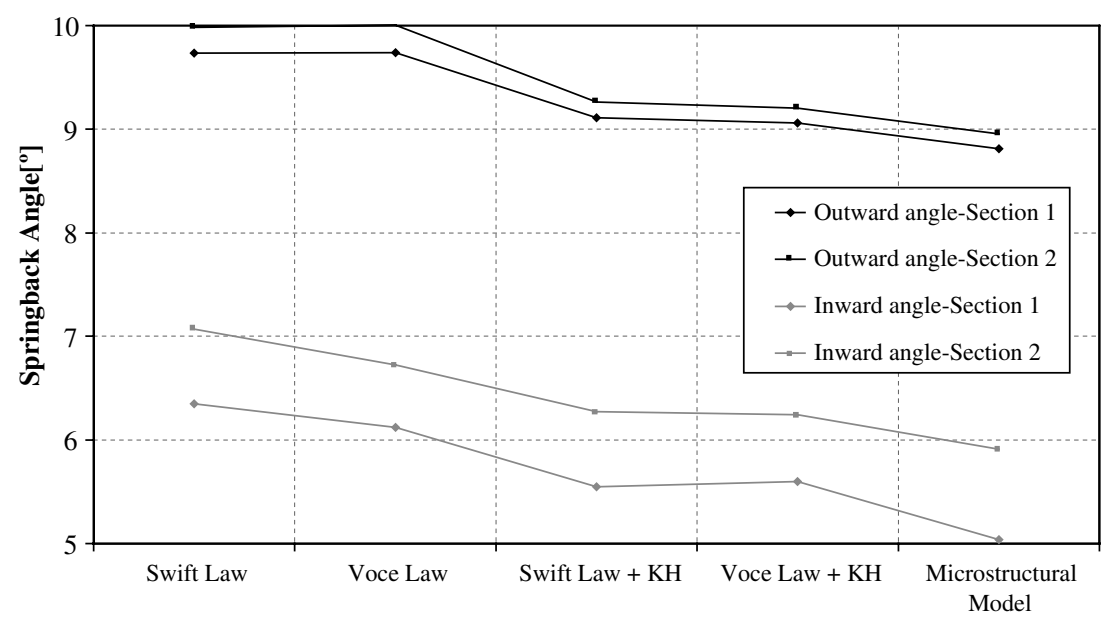

Fig. 19. Influence of the constitutive model on the springback angles of the inward and outward flanges.

for both sections the minimum springback is always attained with the microstructural model. The tests performed only with isotropic hardening lead to small differences in both angles. The introduction of the kinematic work hardening behaviour decreases the springback prediction; the springback angles being $0.8^{\circ}$ less then the angles predicted with the isotropic hardening models. Finally, with the microstructural model these differences are slightly higher (around $1^{\circ}$ ). These differences are related with the bending/unbending strain-path changes that occur during the forming process. The effects of these strain-path changes (mainly Bauschinger effect) in the springback results can not be predicted by the simulations performed only with isotropic work hardening, even for the low level of plastic deformation that occurs in the present forming process $(<15 \%)$.

\section{Acknowledgment}

This work has been carried out as a part of the IMS 199900005 research project Digital Die Design Systems (3DS).

\section{References}

[1] U.F. Kocks, T. Hasegawa, R.O. Scattergood, On the origin of cell walls of lattice misorientations during deformation, Scrip. Metall. 14 (1980) 449-454.
[2] H. Mughrabi, Dislocation wall and cell structures and long-range internal stresses in deformed metal crystals, Acta Metall. 31 (9) (1983) 1367-1379.

[3] T. Hasegawa, T. Yakou, U.F. Kocks, Forward and reverse rearrangements of dislocations in tangled walls, Mater. Sci. Eng. A81 (1986) 189-199.

[4] Y. Strauven, E. Aernoudt, Directional strain softening in ferritic steel, Acta Metall. 35 (1987) 1029-1036.

[5] E.F. Rauch, J.H. Schmitt, Dislocation substructures in mild steel deformed in simple shear, Mater. Sci. Eng. A113 (1989) 441-448.

[6] Y.Z. Liu, D.Y. Dong, K. Yuan, Experimental investigation of microstructural damage in an A-K sheet subjected to two-stage straining, J. Mater. Proc. Technol. 39 (1993) 133-143.

[7] E.F. Rauch, S. Thuillier, Rheological behaviour of mild steel under monotonic loading conditions and cross-loading, Mater. Sci. Eng. A164 (1993) 255-259.

[8] S. Thuillier, E.F. Rauch, Development of microbands in mild steel during cross loading, Acta Metal. Mater. 42 (1994) 1973-1983.

[9] D.V. Wilson, P.S. Bate, Influences of cell walls and grain boundaries on transient responses of an IF steel to changes in strain path, Acta Metall. Mater. 42 (1994) 1099-1111.

[10] E.V. Nesterova, V. Richard, T. Chauveau, TEM examination of the microstructure evolution in the IF steel FeP06 and the bake-hardened steel ZstE180BH under various strain-path changes, LPMTM internal report, May 1999.

[11] J.H. Schmitt, E. Aernoudt, B. Baudelet, Yield loci for polycrystalline metals without texture, Mater. Sci. Eng. 75 (1985) 13-20.

[12] E.V. Nesterova, B. Bacroix, C. Teodosiu, Experimental observation of microstructure evolution under strain-path changes in low-carbon IF steel, Mater. Sci. Eng. A309-310 (2001) 495-499.

[13] E.V. Nesterova, B. Bacroix, C. Teodosiu, Microstructure and texture evolution under strain-path changes in low- 
carbon interstitial-free steel, Metal. Mater. Trans. 32A (2001) 2527-2538.

[14] C. Teodosiu, Z. Hu, Evolution of the intragranular microstructure at moderate and large strains: Modelling and computational significance, in: S.-F. Shen, P.R. Dawson (Eds.), Proc. 5th Int. Conf. Num. Meth. Indust. Forming Process. (NUMIFORM'95), Balkema, Rotterdam, 1995, pp. 173-182.

[15] C. Teodosiu, Materials science input to engineering models, in: S.I. Andersen, et al. (Eds.), Proc. 13th Risø Int. Symp. Mater. Sci.: Modelling of Plastic Deformation and its Engineering Applications, Risø National Laboratory, Roskilde, Danemark, 1992, p. 125.

[16] A. Uenishi, C. Teodosiu, Constitutive modelling of the high strain rate behaviour of interstitial-free steel, Int. J. Plast. 20 (4-5) (2004) 915-936.

[17] P.J. Armstrong, C.O. Frederick, A mathematical representation of the multiaxial Bauschinger effect, C.E.G.B., Report RD/B/N 731, 1966.

[18] J. Lemaitre, J.-L. Chaboche, Mécanique des matériaux solides, Dunod Eds, Paris, 1985.

[19] M.C. Oliveira, J.L. Alves, L.F. Menezes, Improvement of a Frictional Contact Algorithm for Strongly Curved Contact Problems, Int. J. Num. Meth. Eng. 58 (2003) 2083-2101.
[20] L.F. Menezes, C. Teodosiu, Three-dimensional numerical simulation of the deepdrawing process using solid finite elements, J. Mater. Proc. Technol. 97 (2000) 100106.

[21] J.L. Alves, L.F. Menezes, Application of tri-linear and triquadratic 3-D solid finite elements in sheet metal forming process simulation, in: K.-i. Mori (Ed.), Proc. 7th Int. Conf. NUMIFORM 2001, Simulation of Material Processing: Theory, Methods and Applications, Japan, 2001, pp. 639-644.

[22] M.C. Oliveira, J.L. Alves, L.F. Menezes, Springback evaluation using 3-D finite elements, in: Proceedings of the 5th International Conference and Workshop on Numerical Simulation of 3D Sheet Forming Processes, Verification of Simulation with Experiment, NUMISHEET'02, 2002, pp. 189-194.

[23] A. Col, First results of the research project, in: Proceedings of the 22nd International Deep Drawing Research Group Congress and Working Group meeting, Nagoya, Japan, 2002.

[24] S. Bouvier, C. Teodosiu, C. Maier, M. Banu, V. Tabacaru, Selection and identification of elastoplastic models for the materials used in the benchmarks, WP3, Task 1, 18Months Progress Report of the Digital Die Design Systems (3DS), IMS 1999 000051, December 2001. 